\title{
Paris, Arras et la cour : les tapissiers de Philippe le Hardi et Jean sans Peur, ducs de Bourgogne
}

Lorsqu'on recherche des commandes de tapisseries effectuées par les ducs Philippe le Hardi et Jean sans Peur dans les comptes de leurs dépenses quotidiennes, la première mention que l'on trouve le plus souvent ne concerne pas les thèmes figurés sur les tentures, mais le nom, la catégorie professionnelle et le lieu de résidence du fournisseur. Si l'on compare ces données individuelles à la description, souvent prolixe, des produits textiles luxueux qui les suit, on n'est peut-être pas étonné que beaucoup de ces fournisseurs aient bien moins attiré l'attention des chercheurs que les produits qu'ils vendaient à la cour. Dans la bibliographie concernant les tapisseries, l'étude des pièces encore conservées aujourd'hui polarisent l'intérêt des auteurs au détriment d'une analyse approfondie du groupe de ces marchands, même si, depuis quelques années, des historiens d'art, comme Adolph Cavallo, Tom Campbell, Fabienne Joubert et Sophie Schneebalg-Perelman, ont tenté, dans leurs travaux, de saisir qui étaient exactement les fournisseurs de tapisseries des ducs de Bourgogne et de comprendre comment les commandes de ces derniers étaient gérées ${ }^{1}$.

Les recherches, menées durant la même période, sur les fournisseurs de la cour de Bourgogne envisagés d'un point de vue économique, ont mis en

\footnotetext{
*. - Dr Katherine WiLson, Vanbrugh College V/118, Department of History, University of York, Heslington, York, YO10 5DD, Royaume-Uni.

1. - F. Joubert, « Les 'tapissiers' de Philippe le Hardi » dans Artistes, artisans et production artistique au Moyen Âge, t. 3, Fabrication et consommation de l'œuvre. Colloque International. Centre National de la Recherche Scientifique, Université de Rennes II - Haute-Bretagne, 2-6 mai 1983, Paris, 1990, p. 601-608; A.S. Cavallo, Medieval Tapestries in the Metropolitan Museum of Art, New York, 1993; T. CAMPBell, Tapestry in the Renaissance, Art and Magnificence, New York, 2002; S. Schneebalg-Perelman, La tapisserie des Pays-Bas sous les ducs de Bourgogne, Bruxelles, 2003. Pour une vue plus générale: W. BrasSART, Tapisserien und Politik an den Europäischen Höfen, Berlin, 1992; G. Delmarcel, Flemish Tapestries, Londres, 1999 et A. RAPP-BURI et M. STUCKY-SCHÜRER, Burgundische Tapisserien, Munich, 2001. Les acquisitions d'objets de luxe par Philippe le Hardi et Jean sans Peur durant leur principat n'ont été que récemment l'objet d'une attention nouvelle après avoir été longtemps occultées par le long principat de Philippe le Bon. B. Schnerb, Jean sans-Peur: le prince meurtrier, Paris, 2005; Art from the Court of Burgundy: The Patronage of Philip the Bold and John the Fearless 1364-1419, Dijon, 2004.
} 
lumière les liens constants unissant les ducs et ces fournisseurs installés en ville $^{2}$. Pour faire bref, on ne peut plus considérer la cour de Bourgogne comme une entité isolée et on ne peut plus la considérer, nécessairement, comme un élément moteur de la demande de produits de luxe ${ }^{3}$. Graeme Small et Andrew Brown ont récemment insisté sur ces conclusions en affirmant: «La production artistique ne s'est pas développée - et n'aurait pas pu le faire avec les seules commandes de la cour $»^{4}$. Il est clair que, dans les Pays-Bas bourguignons, la clientèle pour les produits de luxe n'était pas seulement aristocratique . Vers 1500 cette région comptait 2,7 millions d'habitants; les contemporains ont fréquemment souligné leur apparente prospérité, par ailleurs largement manifestée par leurs usages vestimentaires ${ }^{6}$. L'un des résultats de cette grande concentration de richesse dans les Pays-Bas, fut le développement d'une demande des consommateurs urbains pour les biens de luxe, et notamment les produits textiles.

À la lumière de cette recherche en plein essor concernant les marchés urbains des produits de luxe et l'impact des commandes de la cour sur la production et la demande urbaines, il semble particulièrement important de centrer l'étude sur les individus dont l'action s'étendait à ces deux sphères: les marchands qui fournissaient la cour en produit de luxe. Peter Stabel est allé jusqu'à considérer que « le rôle de ces individus et les réseaux commerciaux et industriels qu'ils contrôlaient sont des éléments essentiels pour comprendre comment la cour était approvisionnée » ${ }^{7}$. Le propos du présent article sera donc de centrer l'étude sur un groupe particulier, les tapissiers de Philippe le Hardi et de Jean sans Peur. Il s'agira d'étudier les fournisseurs

2. - P. Stabel, «For Mutual benefit? Court and City in the Burgundian Low Countries » dans The Court as a Stage: England and the Low Countries in the later Middle Ages, G. Gunn éd., Londres, 2006, p. 101-117. Son travail est centré sur les principats de Philippe le Bon et Charles le Téméraire. 3. - W. Blockmans, «The Creative Environment: Incentives to and Functions of Bruges Art Production » dans Petrus Christus in Renaissance Bruges an interdisciplinary approach, M.W. Ainsworth éd., Turnhout, 1995, p. 12-15. Cet auteur y affirme: « Ni à Bruges ni dans aucune autre ville des Pays-Bas bourguignons la cour ne fut un facteur décisif, à cause de sa mobilité et de son échelle structurellement réduite. Elle n'aurait jamais pu soutenir le développement de la production de luxe sur une échelle aussi vaste que celle qui est observable à Bruges ».

4. - A. Brown et G. Small, Court and Civic Society in the Burgundian Low Countries c. 14201530, Manchester, 2007, p. 2.

5. - W. Blockmans, « Manuscript Acquisition by the Burgundian Court and the Market for Books in the Fifteenth-Century Netherlands » dans Art Markets in Europe, 1400-1800, M. North and D. Ormrod éd., Aldershot, 1998, p. 7-18.

6. - W. Blockmans et W. Prevenier, The Promised Lands. The Low Countries under Burgundian Rule, 1369-1530, Philadelphie, 1999, p. 151-154. La description sans doute la plus connue est celle du gentilhomme castillan Pero Tafur qui dit de Bruges: «Les gens de cette région du pays sont excessivement fastueux dans leurs vêtements, très extravagants dans leur nourriture et sont très portés sur toutes les sortes de produits de luxe »; M. LETTs, The Travels of Leo of Rozmital through Germany, Flanders, England, France, Spain, Portugal and Italy 1465-1467, Cambridge, 1957, p. 202-203.

7. - P. StaBel, « For Mutual benefit? » (cf. supra n. 2), p. 101-117. 
eux-mêmes, en relation avec les produits qu'ils livraient à la cour, et de relier tout particulièrement leurs activités dans la sphère aulique avec leurs activités urbaines, en préjugeant que les tapissiers de Philippe le Hardi et de Jean sans Peur étaient des hommes d'ambition, particulièrement dans le cadre de leurs communautés urbaines respectives, soucieux d'établir et de maintenir leur réputation professionnelle en fournissant des produits textiles de luxe à la cour, tout en investissant en même temps un marché urbain de consommation en pleine expansion. Je tenterai de montrer aussi que fournir des tapisseries à la cour de Bourgogne était, pour une partie de ces tapissiers, un élément dans une stratégie, " et parfois une stratégie extrêmement risquée », conçue pour maintenir leur réputation et assurer leur survie professionnelle: il était nécessaire pour beaucoup de ces individus d'être étroitement impliqués dans la production et de gérer les commandes de produits textiles de luxe.

Qui étaient donc les tapissiers de Philippe le Hardi et Jean sans Peur? Les informations relatives à ces individus peuvent être trouvées dans les comptes des dépenses ducales de la recette générale des finances ${ }^{8}$. On recense près de cent trente individus, pour les années 1363-1419, qui, soit appartenaient à des catégories professionnelles en relation directe avec la tapisserie, soit intervenaient dans la réparation, le conditionnement ou le transport du matériel. La catégorie professionnelle la plus courante citée au sein de ce groupe est celle de tapissier; la deuxième, moins fréquente, est celle de marchant. Cependant, un certain nombre de ces personnages ressortissaient à plus d'une catégorie professionnelle à la fois et certaines situations, comme celle de valet de chambre ou garde de la tapisserie, conféraient plus de pouvoir et d'influence que d'autres. Par ailleurs, notons que ces gens, dans leur majorité, sont mentionnés, pour la période 1363-1419, comme résidant à Paris et à Arras $^{9}$. Le fait que Paris apparaisse comme la résidence principale de beaucoup de fournisseurs de tapisseries des ducs n'est sans doute pas surprenant, étant donné ce que l'on sait des choix de résidence de Philippe le Hardi et de Jean sans Peur ${ }^{10}$. De plus, Charles VI, Isabeau de Bavière, le duc d'Anjou, le

8. - Les registres cotés Archives départementales de la Côte-d'Or (désormais ADCO) B1413-1603 sont ceux des comptes du receveur général des finances, du trésorier et de l'argentier, contenant les dépenses pour la plupart des exercices annuels, mis à part l'année 1397, et il faut noter que les comptes des années antérieures fournissent moins d'informations. Ces registres et une large partie des pièces comptables sont conservés à Dijon, mais plusieurs registres sont également conservés à Lille, aux archives départementales du Nord (désormais AD Nord), pour le principat de Jean sans Peur (B1878 pour $1405-1406$, B1894 pour $1411-1412$, B1897 pour $1412-1413$ et B1903 pour 14141415). Une partie de cet ensemble est consultable sous forme de microfilms aux archives nationales à Paris, des transcriptions plus tardives se trouvent dans les volumes de la collection de Bourgogne à la Bibliothèque nationale de France à Paris, et des microfilms sont aussi à la Seeley Library de Cambridge.

9. - Malheureusement, les comptes ne précisent pas tous le lieu de résidence des personnages liés au commerce de tapisserie.

10. - Philippe le Hardi a passé la majeure partie de son temps à Paris, ce qui n'est pas surprenant pour un homme qui a joué un rôle majeur au sein du gouvernement royal durant les crises de folie de 
duc de Berry et le duc d'Orléans avaient tous des résidences à Paris ${ }^{11}$. Tous étaient donc des clients potentiels des tapissiers. Paris, toutefois, n'était pas seulement un centre de commercialisation pour une clientèle noble. Avec une population de plus de 80000 habitants à la fin du XIV siècle, la ville comptait aussi de riches familles bourgeoises et un clergé prospère, qui constituaient une possible clientèle pour les tapissiers ${ }^{12}$. Quant à la ville d'Arras, le deuxième lieu de résidence le plus fréquemment cité pour les tapissiers de Philippe le Hardi et Jean sans Peur, bien qu'elle n'ait pas été très souvent un centre pour la cour de Bourgogne, elle était renommée pour la qualité de ses étoffes et était devenue un important centre de production textile de luxe, y compris la tapisserie ${ }^{13}$. Le terme «Arras » est devenu, au cours des siècles, synonyme de textiles de haute qualité et sa renommée était européenne ${ }^{14}$. La population d'Arras comptait de riches marchands d'étoffes et de manieurs d'argent, qui souvent mettaient de grosses sommes à la disposition des villes et des princes ${ }^{15}$.

10. - (suite) Charles VI. Jean sans Peur, lui aussi, privilégia Paris, même si, après le meurtre du duc d'Orléans, les aléas de la situation politique le contraignirent à s'éloigner de cette ville durant certaines périodes (entre 1407 et 1408 et entre 1413 et 1418) et à résider soit en Flandre soit en Bourgogne. Les données concernant leurs résidences se trouvent dans: E. PETIT, Itinéraires de Philippe le Hardi et Jean sans Peur, Ducs de Bourgogne, 1363-1419, Paris, 1888; W. PARAvicini et B. SCHNERB (dir.), Paris, capitale des ducs de Bourgogne, Ostfildern, 2007.

11. - Des extraits de leurs inventaires mobiliers ont été publiés. Voir, J. LABARTE, Inventaire du mobilier de Charles V roi de France, Paris, 1879; J. GUIFFREY, « Inventaire des tapisseries du roi Charles VI vendues par les Anglais en 1422 », Bibliothèque de l'École des Chartes, t. 48, 1887, p. 59-110, 369-444; H. MoranviLlé, «Inventaire de l'orfèvrerie et des joyaux de Louis I ${ }^{\mathrm{er}}$, duc d'Anjou », Bibliothèque de l'École des Chartes, t. 50, 1889, p. 168-179; J. GUIFFrEY, Inventaires de Jean duc de Berry (1401-1416), 2 vol., Paris, 1894; A. Champollion-FigeAC, Louis et Charles ducs d'Orléans leur influence sur les arts, la littérature et l'esprit de leur siècle, Paris, 1884.

12. - R. de LeSPINASSE et F. BonNARdot, Les métiers et corporations de la ville de Paris au XIII siècle, le Livre des métiers d'Étienne Boileau, Paris, 1879; D. CouRTEMANCHE, Euvrer pour la postérité: les testaments parisiens des gens du roi au début du XV viècle, Strasbourg, 1997; B. Bove, Dominer la ville: prévôts des marchands et échevins parisiens (1260-1350), Poitiers, 2000; L. WEIGERT, Weaving Sacred Stories: French Choir Tapestries and the Performance of Clerical Identity, Ithaca, 2004 et L. WEIGERT, « Les tentures de chœur des églises françaises du Moyen Âge à la Renaissance » dans Saints de Chæur. Tapisseries du Moyen Âge et de la Renaissance, L. Weigert, O. Renaudeau, C. Arminjon, et B. Fromaget éd., Paris, 2004, p. 17-40. 13. - K. ZANGGER, Contribution à la terminologie des tissus en ancien français, attestés dans des textes français, provençaux, italiens, espagnols, allemands et latins, Bienne, 1945, p. 14-17 et S. AbrahAM-Thisse, «Les aunes des drapiers au Moyen Âge » dans Cahiers de Métrologie. Actes du $\sigma^{e}$ congrès de métrologie historique, 11-12, Lille, 1992, p. 385; W. KIBLER, G.A. ZINN et L. EARY (dir.), Medieval France: An Encyclopedia, New York, 1995, p. 70.

14. - Les premières références aux draps arrazi/arrazo apparaissent en Italie et une coutume voulait que chaque année, le dimanche de Pâques, le pape offrît à chaque église de Rome une bannière d'arras brodée d'or; cette tradition se poursuivit jusqu' au $\mathrm{XVI}^{\mathrm{e}}$ siècle.

15. - W. Prevenier, « Court and city culture in the Low Countries from 1100 to 1530 » dans Medieval Dutch Literature in its European Context, E. Kooper éd., Cambridge, 1994 p. 15. Cet auteur note que « dès le XIII ${ }^{\mathrm{e}}$ siècle, la ville connaissait même le phénomène de personnes retirées des affaires, les otiose, qui constituaient une catégorie de gens ayant des loisirs et qui, comme les membres de la noblesse de cour, étaient suffisamment riches pour être d'avides consommateurs de biens culturels ». 
Des personnages demeurant à Dijon, Lille, Bruges, Rouvres, Saint-Omer, Hesdin, Lucques, Troyes et Aubeville sont aussi liés au commerce des tapisseries, à leur réparation, à leur entretien et à leur transport. Cependant, quatorze personnes seulement sont mentionnées dans nos sources pour ces différentes localités et sont essentiellement employées par la cour de Bourgogne pour des opérations de réparations, de conditionnement et de transport. Seul Jean Rausart de Lille et Colart d'Auxy d'Hesdin, également cité comme résidant à Arras, appartenant tous deux à la catégorie professionnelle des tapissiers, et Jacques Bise de Bruges, désigné comme marchant, sont indiqués comme vendant des tapisseries à la cour ${ }^{16}$.

Les commandes de tapisseries par la cour ducale ne reflètent donc pas uniquement les choix de résidence des ducs durant la période; c'est une réalité que Peter Stabel a, lui aussi, remarquée en étudiant les achats de produits de luxe durant la période suivante. Il considère que les commandes de la cour (à l'époque de Philippe le Bon et Charles le Téméraire) reposent sur un système faisant entrer en jeu des fournisseurs réguliers, officiers et serviteurs du prince, et les marchés urbains et que « la logique des achats effectués pour la cour est clairement et davantage liée à une hiérarchie des villes marchandes et des centres spécialisés plutôt qu'à l'existence d'une ville de résidence principale $»^{17}$. En étudiant les fournisseurs de tapisseries de Philippe le Hardi et Jean sans Peur de façon approfondie, il apparaît que c'est aussi vrai pour une période antérieure, bien que, comme je le montrerai, être un fournisseur régulier de la cour n'était en rien une situation incontestée.

Durant les principats de Philippe le Hardi et Jean sans Peur, quatre personnages apparaissent comme des fournisseurs réguliers de tapisseries et comme des spécialistes des services liés à cette production: ce sont Jacquet Dourdin, Jean Cosset, Colin Bataille et Pierre de Beaumez. Demeurant à Paris et à Arras, ils sont cités dans les comptes ducaux comme appartenant à plusieurs catégories professionnelles et fournissant à la cour divers produits textiles, des matériaux pour la réparation des textiles et, occasionnellement, d'autres produits de luxe (voir ci-dessous le tableau 1).

16. - ADCO, B1517, fol. 156, B1532, fol 306-308 et B1576, fol. 188-189.

17. - P. StABEL, « For Mutual benefit? » (cf. supra n. 2), p. 39. 


\begin{tabular}{|c|c|c|c|}
\hline Nom & Lieu de résidence & $\begin{array}{c}\text { Catégorie } \\
\text { professionnelle }\end{array}$ & $\begin{array}{l}\text { Produits vendus } \\
\text { à la cour }\end{array}$ \\
\hline Jacquet Dourdin & Paris & $\begin{array}{l}\text { Tapissier, tapissier } \\
\text { sarrasinois, tapissier et } \\
\text { varlet de chambre, } \\
\text { marchand tapissier, } \\
\text { marchand }\end{array}$ & $\begin{array}{l}\text { Tapisserie, tapis de } \\
\text { hautelice, drap de } \\
\text { hautelice, chambre de } \\
\text { hautelice, chambres, } \\
\text { tapis sarrasinois, } \\
\text { tappis, bahuz de } \\
\text { tapisserie, bahuz, tapis } \\
\text { de chapelle, tabel de } \\
\text { autel, pavillions, toille, } \\
\text { toille de Reims, sarge, } \\
\text { sarge de Caen, draps } \\
\text { linges, draps, franges, } \\
\text { franges de soye, } \\
\text { fustanes, camelots, } \\
\text { courtepointe de } \\
\text { baucassin, veluyals, } \\
\text { veluyal sur soye, } \\
\text { quarreaux, coffres, fil } \\
\text { de soye, filde layne }\end{array}$ \\
\hline Jehan Cosset & Arras & $\begin{array}{l}\text { Tapissier, garde de la } \\
\text { tapisserie, varlet de } \\
\text { chambre, marchant }\end{array}$ & $\begin{array}{l}\text { Tapisserie, drap de } \\
\text { tapisserie, chambre de } \\
\text { tapisserie de hautelice, } \\
\text { bahus de tapisserie, } \\
\text { tappis, toille, sarge, } \\
\text { drap de chapelle, } \\
\text { rubans, cordes, canons } \\
\text { d'or, canons d'argent, } \\
\text { fil de layne, fil } \\
\text { d'argent, fil d'Cypre, } \\
\text { fildelin }\end{array}$ \\
\hline Pierre de Beaumez & Paris & $\begin{array}{l}\text { Tapissier sarrasinois, } \\
\text { tapissier, varlet de } \\
\text { chambre, ouvrier de } \\
\text { hautelice }\end{array}$ & $\begin{array}{l}\text { Tapisserie, tapis } \\
\text { sarrasinois, drap de } \\
\text { hautelice, tapis de } \\
\text { hautelice, tapis, } \\
\text { marchepieds, banquier, } \\
\text { quarreaux, toille, } \\
\text { cordes }\end{array}$ \\
\hline Nicolas/Colin ${ }^{18}$ Bataille & Paris & $\begin{array}{l}\text { Tapissier, marchant, } \\
\text { marchant tapissier, } \\
\text { marchant de tapisserie } \\
\text { sarrasinois }\end{array}$ & $\begin{array}{l}\text { Tapis, sarge, sarge de } \\
\text { Caen, chambre, toille, } \\
\text { quarreaux, } \\
\text { marchepied, says, } \\
\text { cordes, franges }\end{array}$ \\
\hline
\end{tabular}

En outre, ces hommes étaient employés pour le transport de tapisseries et d'autres produits textiles de luxe. En 1389, Jacquet Dourdin mena, d'Arras à Dijon, un grand drap de hautelice représentant l'histoire de sainte Catherine; un type de mission dont il fut encore chargé en 1394 et $1397^{19}$. Pierre de Beaumez fut chargé de transporter des quarreaux de Paris à Arras, et d'effec-

18. - Colin est le diminutif de Nicolas.

19. - ADCO, B1503, fol. 63 et B1511, fol. 108 . 
tuer aussi plusieurs déplacements de Paris à Conflans en 1387 et $1395^{20}$. Ces personnages eurent également à assurer des travaux de réparation. Jean Cosset fut payé à trois reprises pour réaliser des bordures pour des sarges, tapis et drap de hautelice, et une fois, en 1394, pour teindre des cordes et rubans $^{21}$. Nicolas Bataille, quant à lui, est le seul fournisseur de tapisseries important que je n'ai pas encore trouvé mentionné dans une opération de réparation, de modification, de conditionnement ou de transport de produits textiles.

Il est clair que ces quatre personnages étaient capables de fournir une large gamme de textiles de luxe et d'assurer aussi des services à la cour de Bourgogne. Il semble qu'avoir accès à une grande variété de produits et de services était un élément-clé qui attirait la clientèle ducale. Mais qu'est-ce que leurs concurrents, les cent vingt-six autres individus recensés, trouvaient dans le fait de fournir la cour ducale? Quand on étudie le groupe des autres fournisseurs de tapisseries, il est frappant de constater que la majorité des tapissiers de Philippe le Hardi et Jean sans Peur apparaissent moins de cinq fois dans la comptabilité. En fait, la majeure partie d'entre eux livre des produits ou assurent des services en une occasion seulement, et ce fait est vrai pour les autres fournisseurs mentionnés comme demeurant à Paris, à Arras ou ailleurs. Par exemple, Étienne Trouchet, tapissier et marchant de Paris, fut payé 26 fr. 15 s. t. en 1394 pour divers produits et services ${ }^{22}$. Les documents comptables précisent qu'il livra un rideau pour une chambre bleue de même qu'une couverture de lit, un ciel et une dossière portant des images de moutons brodés pour une autre chambre. Il fut aussi payé pour réparer des rideaux, pour border de cuir la chambre aux moutons et pour livrer des cordes rouges, probablement utilisées pour la réalisation de la dite chambre. Cinq ans plus tard, ses services furent de nouveau sollicités, pour fournir, cette fois, une livre de soie et d'or de Chypre, du fil d'Arras, et 24 aunes de ruban pour la réparation de tapis ${ }^{23}$. Pour ces produits, il reçut $72 \mathrm{fr} .8 \mathrm{~s} .4 \mathrm{~d}$. t. Des cas de figure similaires apparaissent pour d'autres fournisseurs de tapisseries d'Arras. Jean Cosset est le seul Arrageois mentionné avec une réelle régularité dans la comptabilité ducale, les dix-neuf autres personnes apparaissant moins de sept fois durant les principats de Philippe le Hardi et de Jean sans Peur. Jean Armory, tapissier, est cité pour avoir passé vingt jours à réparer des tapisseries, tandis que Jean des Capelles, lui aussi tapissier, est payé en 1412 pour plusieurs tapis achetés pour être présentés en don à divers gentilshommes anglais ${ }^{24}$. Bien que de telles commandes aient pu être lucratives,

20. - ADCO, B1466, fol. 34 et B1469, fol. 26.

21. - ADCO, B1481, fol. 27 et B1511, fol. 102.

22. - ADCO, B1501, fol. 61.

23. - ADCO, B1517, fol. 125.

24. - ADCO, B1570, fol. 218. 
elles n'étaient pas suffisantes pour que ces tapissiers en vivent. Pour prendre un exemple, le paiement des deux commandes mentionnées équivalait à 159 jours de travail d'un maître maçon ${ }^{25}$. Pourquoi donc, alors, fournir la cour de Bourgogne si les commandes étaient si limitées, le marché étant dominé, comme nous l'avons vu, par nos quatre fournisseurs réguliers ? Et comment était-il possible à ces cent vingt-six individus de survivre, et à ces quatre hommes de monopoliser les ventes de tapisseries à la cour, alors même qu'il est clair que la concurrence existait de la part d'autres personnages capables de procurer des produits et des services du même type ? On trouve la réponse à ces questions en étudiant la stratégie qu'ils ont appliquée pour maintenir leur réputation et assurer leur survie professionnelle en constituant et entretenant de puissants réseaux tant à la cour qu'à la ville.

Jacquet Dourdin réussit particulièrement bien à entretenir ses réseaux à Paris. Non seulement ce personnage apparaît fréquemment dans la comptabilité bourguignonne du temps de Philippe le Hardi, mais il s'est affirmé aussi comme fournisseur de plusieurs autres personnages de premier plan, en particulier Charles VI, son frère Louis, duc d'Orléans, et Isabeau de Bavière. La situation de Dourdin, qui était valet de chambre du roi et du duc de Bourgogne, lui assurait une position privilégiée en tant que serviteur, tant à la cour royale qu'à la cour ducale, une position dont bénéficiaient deux des plus actifs fournisseurs de tapisseries de la cour. En 1396, il reçut 18001 . du duc d'Orléans pour trois tappis de hautelice de fins fils d'Arras, ouvréz a or de Chippre, les deux premières représentant les articles du Credo avec douze prophètes et les douze apôtres et la troisième représentant le Couronnement de la Vierge ${ }^{26}$. En 1399, il est mentionné comme payé par ordre d'Isabeau de Bavière pour un tappis de fil d'Arras représentant la destruction de Troie ${ }^{27}$. En tant que résident parisien, il était bien placé pour offrir en permanence ses services au roi, à la reine, au duc d'Orléans et à Philippe le Hardi. En outre, sa position de valet de chambre du roi de France et du duc de Bourgogne lui conférait un avantage certain sur les autres fournisseurs et prestataires de services de Paris. Son aptitude à procurer à la cour des matériaux de luxe était un atout qui assura sa suprématie pour près de vingt ans.

Nicolas, alias Colin, Bataille était un autre de ces personnages capables de fournir une grande variété de produits textiles de luxe à la cour et qui entretenaient aussi un réseau de relations à Paris ${ }^{28}$. La réussite de son entreprise de

25. - Les gages journaliers moyens d'un maître maçon à Bruges étaient de 10 gros de Flandre durant la période 1370-1420. J.-P. Sosson, Les travaux publics de la ville de Bruges XIV -XVe siècles, Bruxelles, 1977, p. 303.

26. - Baron de Joursanvault, Catalogue analytique des archives de M. le Baron de Joursanvault, Paris, 1838 , p. 156.

27. - Paris AN, KK 42, fol. 223-224.

28. - Tout comme Jacquet Dourdin, il est cité dans presque tous les ouvrages concernant la tapisserie parus au XIX ${ }^{\mathrm{e}}$ siècle. Voir J. GUIFFREY, Nicolas Bataille tapissier parisien du XIVe siècle, auteur de l'Apocalypse d'Angers, Paris, 1877. 
captation des commandes de nombreux clients de haut-rang, vint probablement du fait qu'il pouvait leur fournir plus que des tapisseries. Bien que mentionné comme marchant de tapisserie, marchant de tapisserie sarrasinoise, marchant et tapissier, il vendit essentiellement des sarges à Philippe le Hardi durant la période 1369-1395 29 . Ces sarges étaient de différentes couleurs et de différentes nuances et servaient à la réalisation de chambres constituées de différents types d'étoffes. En 1372, il fut payé pour dix pièces de sarges vermeilles destinées à compléter une chambre de camocaz ${ }^{30}$. En une autre occasion, en 1388, six autres sarges vermeilles lui furent achetées, destinées cette fois à une chambre de veluyau en graine a long poil ${ }^{31}$. Il pouvait toutefois fournir également des tapisseries au duc lorsqu' on le lui demandait. En 1395, il vendit une chambre de tapisserie de fin fille d'Arras de couleur bleue avec une couverture de sarge avec dais ${ }^{32}$. Mais en aucune façon il ne comptait sur la seule clientèle du duc de Bourgogne pour soutenir son négoce. Un autre de ses clients de haut-rang n'était autre que le roi Charles $\mathrm{V}$, dont les gens de finances, en 1376, connaissaient Nicolas Bataille en tant que marchand de tapis sarrasinois ${ }^{33}$. À ce royal client, il fournit encore douze larges tapis, douze couvertures de bancs et seize sarges. Un autre de ses riches commanditaires était Amédée VI, comte de Savoie, à qui il vendit une chambre ornée d'aigles et de nouds ${ }^{34}$. En 1373, il est cité comme valet de chambre de Louis I ${ }^{\mathrm{er}}$, duc d'Anjou. En 1389, on le trouve même passant, en compagnie de Jacquet Dourdin, un contrat avec Louis de France, futur duc d'Orléans, pour la réalisation d'une tapisserie représentant les joutes de Saint-Denis ${ }^{35}$. Finalement, on le retrouve avec le titre de valet de chambre du duc de

29. - Les sarges fournies à la cour de Bourgogne étaient nettement moins coûteuses que les draps de hautelice, tapisseries et pièces de velours.

30. - ADCO, B4418, fol. 22. Le camocaz était une soierie de luxe dans le genre du damas et pouvait être utilisé aussi bien pour des vêtements que pour des tentures. K. ZANGGER, Contribution à la terminologie (cf. supra n. 13), p. 40-41.

31. - ADCO, B1486, fol. 29. Le terme « graine » renvoie à un type de teinture utilisé pour les vêtements, notamment écarlate, tandis que le terme « long poil » semble faire référence à l'épaisseur du velours. G. DE PoERCK, La draperie médiévale en Flandre et en Artois: technique et terminologie, t. 2, Bruges, 1951, p. 100 et J. Munro, «The Medieval Scarlet and the Economics of Sartorial Splendour » dans Cloth and Clothing in Medieval Europe : Essays in Memory of E.M. Carus-Wilson, N.B. HARTE et K.G. Ponting éd., London, 1983, p. 13-70.

32. - ADCO, B1486, fol. 52.

33. - W.G. Thompson donne une référence de la British Library (Add. Ch., n 2772). Nicolas Bataille procura au duc d'Anjou des tentures représentant l'Arbre de Vie, avec l'image du Crucifix et divers prophètes dans les branches de l'arbre. British Library, Add. Ch., n 2772. En 1399, il lui vendit encore une tenture semée de licornes et d'autres animaux. British Library, Add. Ch., n 2734. Toutes ces références sont également données par: W.G. THOMPSON, A History of Tapestry from the Earliest Times until the Present Day, Londres, 1930.

34. - Ibid., p. 63.

35. - Cette tenture était probablement commandée pour commémorer les fêtes organisées à SaintDenis en cette même année 1389 et au cours desquelles Louis de France et Louis d'Anjou furent tous deux adoubés. 
Bourgogne $^{36}$. Les comptes ducaux angevins mentionnent de nombreux paiements à lui faits pour fourniture de produits semblables à ceux qu'il procurait à la cour de Bourgogne, comme des chambres, des draps, des sarges de différentes couleurs. Il est toutefois plus connu pour la réalisation de la tapisserie de l'Apocalypse commandée par le duc d'Anjou en 1377, que l'on peut toujours admirer au château d'Angers ${ }^{37}$.

On ne sait malheureusement que peu de chose, à l'heure actuelle, sur les autres clients de ces personnages. Il est clair, toutefois, que Jacquet Dourdin et Nicolas Bataille ne comptaient ni sur le patronage d'un seul client ni sur leur seule aptitude à vendre des tapisseries pour survivre professionnellement. Il est même possible que ce soit la possibilité de fournir plus d'un type de produits et d'attirer d'autres clients qui leur assura une prééminence parmi les fournisseurs de la cour de Bourgogne. On ne saurait omettre aussi qu'on trouve au moins trente-trois autres individus dans les comptes ducaux du temps de Philippe le Hardi et de Jean sans Peur qui étaient en mesure de vendre des tapisseries ou d'assurer des services liés à la tapisserie, comme la réparation par exemple, et qui, à l'occasion, étaient également aptes à assurer ces services à plus d'une cour princière. Perrenelle de Crepon, qui était, elle aussi, clairement liée à la cour du roi de France - elle est citée comme tapissier du roy dans la comptabilité bourguignonne -, fournit des sarges à Philippe le Hardi en $1374^{38}$. En outre, à un niveau inférieur à celui de Jacquet Dourdin et Colin Bataille, il y avait une vive concurrence, et dans le cas où ils se seraient trouvés dans l'incapacité de faire face à une commande ducale, il n'est pas douteux que d'autres professionnels auraient été prêts et décidés à prendre leur place.

Paris n'était pas le seul centre où les tapissiers de Philippe le Hardi et Jean sans Peur pouvaient exercer leur activité. Arras était également un lieu parfait pour des entrepreneurs ambitieux, désireux de s'affirmer à la ville et à la cour, tirant parti d'une production textile en plein développement et internationalement réputée et d'une pléthore de clients potentiels dans la ville elle-même ${ }^{39}$.

36. - W.G. ThOMPson, A History of Tapestry (cf. supra n. 33), p. 63.

37. - L'ensemble, dans son état originel, comptait sept pièces et probablement quatre-vingtquatre scènes. 50 fr. furent payés à Hennequin de Bruges, alias Jan Bondol ou Bandol, peintre et valet de chambre de Charles $\mathrm{V}$, pour la réalisation des cartons à partir desquels la série fut faite. R. Planchenault, L'Apocalypse d'Angers et les débuts de la tapisserie historiée, Paris, 1966; F. JOUBERT, «L'Apocalypse d'Angers et les débuts de la tapisserie historiée », Bulletin monumental, 1981 ; P. AuZAS, L'Apocalypse d'Angers : Chef-d'œuvre de la tapisserie médiévale, Paris, 1985. Sur la coopération entre les différents corps de métier à Bruges à la même époque, voir: P. STABEL, « Organisation corporative et production d'œuvres d'art à Bruges à la fin du Moyen Âge et au début des Temps modernes », Le Moyen Âge, t. 113, 2007, p. 91-134.

38. - ADCO, B1444, fol. 53.

39. - Pero Tafur passa aussi par Arras en 1432 et décrivit la ville comme « ...un lieu plaisant et très riche, spécialement en raison de ses draps et de toutes sortes de tapisseries ». M. LETTS, The Travels of Leo of Rozmital (cf. supra n. 6), p. 201-202. 
Bien que les auteurs de travaux traitant de la tapisserie répètent, de façon routinière, l'affirmation selon laquelle les fournisseurs de tapisseries devaient exercer plusieurs professions, ils n'ont guère tenté de savoir pourquoi. Cependant, comme je crois l'avoir démontré ici, la majorité des tapissiers sont mentionnés moins de cinq fois dans la comptabilité ducale et, en conséquence, les commandes du prince pour la majeure partie de ces tapissiers, étaient, au mieux, sporadiques. Même pour les fournisseurs réguliers de la cour de Bourgogne le succès n'était jamais assuré, étant donné la concurrence que représentaient les autres. On ne devrait donc pas être surpris de les voir pratiquer d'autres métiers et de proposer d'autres produits. Il fallait à l'homme médiéval, et aussi à la femme, diverses activités et diverses spécialités pour survivre professionnellement. Gervase Rosser et Peter Stabel ont montré tous deux la relative facilité avec laquelle certains personnages pouvaient cumuler de multiples appartenances à des corps de métier ou avoir «plusieurs identités » et donc plusieurs positions professionnelles ${ }^{40}$. En étudiant les carrières et l'arrière-plan socioprofessionnel de nombreux tapissiers en relation avec la cour de Bourgogne, il apparaît clairement que ces individus tiraient un grand avantage de leur «pluralité d'identités », afin de consolider leur ascension sociale et d'assurer leur survie financière dans la ville. Jean Cosset, un autre des principaux tapissiers de la cour de Bourgogne, a été identifié par Jean Lestoquoy comme un membre d'une famille arrageoise prospère et influente, la première attestation du nom Cosset remontant à $1217^{41}$. Lestoquoy a reconstitué cinq générations de cette famille, repérant des Cosset apparaissant à travers tout le XIII ${ }^{\mathrm{e}}$ siècle, pratiquant le prêt d'argent et contractant des alliances matrimoniales avec des familles plus puissantes que la leur ${ }^{42}$. Quand on arrive au Jean Cosset, fournisseur de tapisseries de la cour de Bourgogne dans les années 1380 et 1390, il apparaît comme un homme aux activités multiples: aubergiste et pas moins de cinq fois échevin, effectuant aussi plusieurs transactions profitables dans le cadre du commerce du vin, et mentionné comme valet de chambre et tapissier du duc. En tant que membre d'une famille qui avait saisi plusieurs occasions d'ascension sociale, il pouvait considérer que la chance de fournir la cour pouvait être, elle aussi, une opportunité souhaitable pour poursuivre et maintenir cette ascension.

40. - G. Rosser, « Crafts, Guilds and the Negotiation of Work in the Medieval Town », Past and Present, t. 154, 1997, p. 8; P. STABEL, « Guilds in Late Medieval Flanders: Myths and Realities of Guild Life in an Export Orientated Environment », Journal of Medieval History, t. 30, 2004, p. 193. Cet auteur note: «si l'on observe de plus près les réalités de l'appartenance aux guildes [...] on ne peut faire autrement que de remarquer que cette appartenance n'était pas un phénomène exclusif dans la ville de la fin du Moyen Âge et que les artisans appartenaient souvent à plusieurs guildes en même temps ».

41. - J. LeStOCQUOY, « Financiers, courtiers, hautelisseurs d'Arras aux XIII et XIV ${ }^{\mathrm{e}}$ siècles », Revue Belge de Philologie et d'Histoire, Bruxelles, 1938, p. 919-920. Voir aussi ID., Les dynasties bourgeoises d'Arras du XI ${ }^{e}$ au XV $V^{e}$ siècle, Arras, 1952 et ID., Deux siècles de l'histoire de la tapisserie (1300-1500). Paris, Arras, Lille, Tournai, Bruxelles, 1978.

42. - J. LESTOCQUOY, « Financiers, courtiers » (cf. supra n. 41), p. 919. 
Plusieurs des tapissiers apparaissant moins de cinq fois dans la comptabilité bourguignonne étaient aussi liés entre eux au sein d'un réseau d'affaires à Arras. Huart Wallois, qui est mentionné quatre fois en tant que tapissier dans les documents comptables, entre 1383 et 1407, fournit plusieurs pièces d'étoffe à la cour ducale. En 1383, il fut payé à deux reprises, la première pour dix pièces de soie blanche et verte pour la confection des tapis d'une chambre et la seconde pour la vente de rubans, peut-être pour border les tapis de cette chambre ${ }^{43}$. On le trouve une fois encore livrant huit tapis de hautelice ornés de marguerites ${ }^{44}$. Le dernier paiement à lui fait date de 1407 et concerne la livraison de quatre bahus de tapisserie armoriés des armes de madame la duchesse ${ }^{45}$. Jean Lestocquoy note que, tout comme Jean Cosset, il fut échevin, renforçant sa position par la possession de nombreux biens immobiliers $^{46}$. En 1375, Huart Wallois, qui avait été échevin en 1373 et était membre de la Confrérie Notre-Dame, était aussi impliqué dans le commerce du drap et du vin ${ }^{47}$.

Des alliances matrimoniales organisées avec soin étaient indubitablement l'une des clés du maintien d'entreprises professionnelles fructueuses, en particulier si elles permettaient de s'unir à des partenaires entretenant des relations commerciales similaires avec une clientèle bien établie ${ }^{48}$. Huart Wallois saisit la chance de contracter un mariage stratégique avec Marguerite Boursette, fille d'un autre fournisseur de tapisseries de la cour de Bourgogne, Vincent Boursette ${ }^{49}$. Son beau-père avait assuré des services liés à la tapisserie pour la cour de Philippe le Hardi en 1374 et 1376, tout d'abord en conditionnant des tentures en vue de leur transport et en effectuant le transport d'Arras à Montbard, puis en fournissant du fil pour la réparation de plusieurs tapisseries $^{50}$. On ne sait que peu de choses de lui, sinon que sa fille épousa le dit Huart Wallois et qu'il devint, lui aussi, échevin d'Arras en $1375^{51}$. Pierre Le Conte, en 1385, vendit au duc de Bourgogne une tapisserie représentant l'histoire de saint Antoine pour un prix de $15001 .^{52}$. Bien qu'il n'apparaisse qu'une seule fois comme fournisseur de tapisseries de la cour ducale, il fut

\footnotetext{
43. - ADCO, B1461, fol. 83.

44. - ADCO, B1479, fol. 49. Les marguerites étaient l'un des emblèmes de Marguerite de Male, épouse de Philippe le Hardi.

45. - ADCO, B1556, fol. 106.

46. - J. LESTOCQUOY, Les dynasties bourgeoises (cf. supra n. 41), p. 48.

47. - J. LeSTOCQUOY, « Financiers, courtiers » (cf. supra n. 41), p. 913-914.

48. - K. Brosens, « New data on Albert Auwerx. Toward a Conceptual Framework of the Entrepreneurial Strategies Devised by Brussels Tapestry Producers, 1600-1700 ». Dans cet article inédit, l'auteur a montré que la stratégie matrimoniale visant à établir des réseaux utiles et à se créer une clientèle était aussi importante au XVII ${ }^{\mathrm{e}}$ siècle qu'elle l'était auX $\mathrm{XIV}^{\mathrm{e}} \mathrm{et} \mathrm{XV}^{\mathrm{e}}$ siècles.

49. - Ibid., p. 48.

50. - ADCO, B1384 (liasse), B1444, fol. 49 et 51; B1445, fol. 64.

51. - J. LestOCQUOY, Les dynasties bourgeoises (cf. supra n. 41), p. 48.

52. - Ibid., p. 47.
} 
échevin d'Arras six fois, de 1392 à 1405, et possédait sept biens immobiliers dans la ville ${ }^{53}$. Tout comme Jean Cosset et Huart Wallois il ne dépendait pas que de la tapisserie et faisait de substantiels profits en tant que marchand de vin.

André de Monchy, un autre habitant d'Arras, est mentionné dans la comptabilité ducale au moins quinze fois entre 1390 et 1401 . Les comptes le citent comme tapissier, comme marchant, ou comme marchant de tapisserie. Sa famille émerge en 1295, quand on trouve un Jean de Monchy et un Stéphane de Monchy impliqués dans un prêt d'argent à la ville de Bruges ${ }^{54}$. Jean de Monchy prêta la somme de 20001 . et Stéphane $11401 .{ }^{55}$. Suivant un modèle semblable à celui des familles engagées dans la fourniture de tapisseries et d'autres produits à la cour de Bourgogne, leurs descendants furent investis de charges municipales dans le courant du XIV ${ }^{\mathrm{e}}$ siècle. Il est possible que ce soit le père d'André de Monchy, fournisseur de la cour, qui ait été échevin entre 1314 et 1355, apparaissant aussi comme receveur d'Artois de 1319 à $1329^{56}$. Un autre de Monchy fut échevin entre 1393 et 1428 . Un autre rapprochement avec les personnages mentionnés plus haut réside dans le fait qu'il a été sans doute lié au commerce du vin, ayant été fermier de la maltôte du $\operatorname{vin}^{57}$.

Les produits qu'André de Monchy se procurait pour les livrer à la cour étaient de haute qualité, certains devant servir à des dons. La première pièce qu'il acquit ainsi était un tapis représentant Perceval le Gallois, offert en 1390 au duc d'York, et pour lequel il reçut un remboursement de $150 \mathrm{fr}^{58}$ On fit encore appel à ses services pour fournir plusieurs tentures qui furent données à des membres de la cour d'Angleterre en 1393 et 1397. Deux draps d'or avec des figures de dames et représentant l'histoire de Plaisance furent expédiés en Angleterre par les soins de Jean Le Cambier ${ }^{59}$. En 1397 un tapis avec fil d'or représentant la Trinité, pour lequel André de Monchy avait payé 6751 ., fut cette fois destiné au roi d'Angleterre lui-même ${ }^{60}$. Il se procura aussi, en 1395, 1400 et 1401, plusieurs luxueuses chambres réalisées à partir de plusieurs matériaux et représentant différents sujets. En 1395, une chambre de vermeille à la devise du comte de Nevers est mentionnée comme ayant coûté 715 fr. 2 s. 6 d. t. ${ }^{61}$ Cet ensemble consistait en tapis pour les murs,

\footnotetext{
53. - Ibid., p. 47.

54. - J. LESTOCQUOY, « Financiers, courtiers » (cf. supra n. 41), p. 915.

55. - Ibid. p. 915.

56. - Ibid.

57. - Ibid.

58. - ADCO, B1495, fol. 72. La somme représente 240 jours de salaire pour un maître maçon de Bruges.

59. - ADCO, B1500, fol. 137.

60. - ADCO, B1511, fol. 109.

61. - Ibid. Cette somme équivaut exactement à trois ans de salaire d'un maître maçon.
} 
plus une sarge de lit, un banquier, un tapis pour la couchette et un marchepied, le tout pour la dite chambre ${ }^{62}$. En outre, il comportait un banquier et un marchepied destinés à une autre chambre du comte de Nevers ${ }^{63}$. En 1400, il fournit à la cour, pour la somme de 300 écus d'or, une autre chambre de hauteliche qui fut donnée à un chambellan du duc ${ }^{64}$. Une chambre de tapisserie semez d'arbres fut le dernier produit livré par André de Monchy, en 140165; elle comportait une sarge pour le lit, ornée de motifs de feuillage en soie d'or et d'argent, des tapis de hauteliche pour les murs, ciels et dossiers avec des franges de soie, un drap pour la couchette et une couverture de banc ${ }^{66}$.

Désigné comme tapissier et marchant dans la comptabilité ducale, Michel Bernart, demeurant à Arras, était un personnage qui tirait profit du commerce du vin et, tout à la fois, pouvait fournir des tapisseries à Philippe le Hardi. Il est surtout connu pour avoir reçu commande de la tapisserie de la bataille de Roosebecke, qui coûta 3600 fr. en $1386^{67}$. La même année, il livra au duc une tapisserie représentant Firebrat d'Alexandre qui fut par la suite offerte à Pierre, comte de Genève ${ }^{68}$. En 1390 et 1393, il fut encore payé pour avoir procuré des tapis destinés à être offerts au duc d'York et, à l'occasion des étrennes du jour de l'An, à Richard II et à la duchesse de Gloucester; ces tentures représentaient l'histoire de Clovis et l'histoire de Notre Dame ${ }^{69}$.

En vérité, fournir des produits textiles de haute valeur à la cour de Bourgogne se révèle une claire stratégie, pour un certain nombre de tapissiers d'Arras, destinée à asseoir la renommée de leur famille et de leur production. Ce motif explique pourquoi cent vingt-six autres tapissiers s'efforcèrent de recevoir une commande ducale, même si elle devait être la première et la dernière. Ce qui est clair lorsqu'on étudie ces tapissiers arrageois de façon plus approfondie, est que nombre d'entre eux ne pouvaient compter sur la seule clientèle ducale pour s'assurer des revenus suffisants, ce qui était aussi vrai pour les fournisseurs les plus importants que pour les moins importants.

\footnotetext{
62. - Ibid

63. - Ibid.

64. - ADCO, B1519, fol. 232. Cette chambre était moins coûteuse que les précédentes si on en rapporte le prix au salaire journalier d'un maître maçon, puisqu'elle ne représente que 135 journées de travail.

65. - ADCO, B1526, fol. 249.

66. - Ibid. La valeur de l'ensemble s'élève à 1500 fr., soit 2400 jours de travail pour un maître maçon.

67. - AD Nord, B4075, fol. 64. Sur cette somme, 300 fr. furent payés pour la réalisation des cartons. 68. - ADCO, B1466, fol. 16. Fierabras d'Alixandre, roi sarrasin, fils de l'amiral Balant, baptise Florent, voir A. MoISAN, Répertoire des noms propres de personnes et de lieux cités dans les Chansons de Geste françaises et les auvres étrangères dérivées, t. II, Genève, Droz, 1986, p. 201. 69. - ADCO, B1495, fol. 72 et B1500, fol. 137. J. HiRSCHBIEGEL, Étrennes. Untersuchungen zum höfischen Geschenkverkehr im spätmittelalterlichen Frankreich der Zeit König Karls VI. (13801422), Munich, 2000 et B. BuetTner, « Past Presents: New Year's Gifts at the Valois Courts, ca. 1400 », The Art Bulletin, 83, 2001, p. 598-625.
} 
Jacquet Dourdin et Nicolas Bataille eux-mêmes étaient dans la nécessité de rechercher des commandes d'autres clients à Paris. Et hors des possibles réseaux de la cour, il fallait, et il était même indispensable, d'avoir accès à d'autres activités lucratives. Pour Jean Cosset, André de Monchy, Michel Bernart, Pierre Le Conte et Huart Wallois, le commerce du vin, le prêt d'argent, les charges municipales permettaient de faire de bonnes affaires pour maintenir leur réputation et assurer leur survie professionnelle. Si rentables que pouvaient être les commandes du duc de Bourgogne, d'autres clients étaient nécessaires, d'autant que le volume des achats de la cour était loin de suffire à vider le marché des tapisseries, sarges, camocaz et damas disponibles. Bien qu'il ne soit pas dans le propos de cet article de traiter des autres clients des produits proposés par les tapissiers de Philippe le Hardi et Jean sans Peur, il n'est guère douteux que les personnages étudiés ici aient aussi accepté des commandes de clients qui n'étaient ni rois, ni ducs, ni princes ${ }^{70}$. Ainsi donc, pour rester efficaces, les tapissiers devaient s'assurer que leur marchandise et leur réputation touchaient autant de clients potentiels que possible. Pour un artisan ou un marchand,

« la préoccupation de loin la plus importante... était l'établissement d'une bonne réputation personnelle. Tant qu'on ne pouvait revendiquer une bonne renommée, il était impossible d'obtenir un crédit personnel - ce crédit personnel sans lequel on ne pouvait survivre dans l'économie urbaine $»^{71}$.

Procurer des produits textiles de qualité à la cour de Bourgogne « même une seule fois » était un moyen de gagner une bonne réputation. Devenir fournisseur de la cour et y obtenir une position étaient peut-être un autre aspect de la stratégie mise en œuvre par les tapissiers pour se distinguer des autres individus impliqués dans le même commerce et prendre l'avantage sur eux.

Des précisions données dans la comptabilité ducale indiquent que certains tapissiers bénéficiaient d'une position privilégiée par rapport à d'autres fournisseurs en étant titulaire d'un office de l'hôtel, tel celui de valet de chambre et de garde de la tapisserie. Sur près de cent trente personnages impliqués dans la fourniture, l'entretien et la réparation des tapisseries, moins de dix reçurent le titre de valet de chambre. Sans qu'on en soit surpris, plusieurs de ceux-ci apparaissent dans les comptes parmi les fournisseurs fréquents. Des quatre plus importants, trois étaient valets de chambre: Jacquet Dourdin, Jean Cosset et Pierre de Beaumez. Jacquet Dourdin portait le même titre dans l'hôtel du duc Louis I I' d'Anjou. Porter ce titre présentait d'incontestables avantages pour des marchands qui, sans nul doute, souhaitaient accroître et resserrer les liens qui les unissaient à la cour. Hugo Van der Velden a souligné

70. - Ces autres clients sont étudiés dans ma thèse de doctorat Courtly and Urban Tapestries of the Burgundian Dominions c. 1363-1500 (à paraître chez Brepols).

71. - G. Rosser, « Crafts, Guilds and the Negotiation of Work», (cf. supra n. 40), p. 9. 
que l'octroi de cette position était pour le duc non seulement un moyen d'avoir sous la main un fournisseur digne de confiance, mais aussi une manière de manifester sa faveur ${ }^{72}$. Bien qu'il y ait eu de nombreux varlets de chambre à la cour de Bourgogne, une telle fonction pouvait représenter une position puissante et lucrative et aussi un premier échelon dans une ascension vers des offices plus élevés. Être investi d'une telle charge signifiait en principe être le premier sur la liste pour l'octroi d'une belle commande de drap de hautelice, tapisserie, sarges, soieries ou velours.

L'office de garde de la tapisserie de l'hôtel ducal semble avoir également conféré des privilèges et des obligations à son titulaire. Il paraît avoir été relativement exclusif puisqu'on ne repère que huit personnes l'ayant assumé durant les principats de Philippe le Hardi et Jean sans Peur. Le titre apparaît pour la première fois en 1388 et est alors porté par Jean Le Cambier, mentionné comme valet de chambre et garde de la tapisserie. Il conserva cette charge lorsque Jean sans Peur succéda à son père ${ }^{73}$. À la mort de Philippe le Hardi c'est lui qui fut chargé de dressé l'inventaire de toutes les chambres, tapisseries et autres choses, de même que de toute la vaisselle et des pennons et bannières ayant appartenu au duc défunt ${ }^{74}$. Sa femme et lui bénéficièrent d'une queue de vin vieux à la fin des années 1390, en récompense de leurs services ${ }^{75}$. Jean de Neufport put, lui aussi, conserver sa charge de garde de la tapisserie après la mort de Philippe le Hardi $^{76}$. Jean Cosset en fut aussi l'un des titulaires sous le principat de Philippe le Hardi ${ }^{77}$. Sous Jean sans Peur, on trouve deux autres personnages ayant occupé la fonction: Jean Prevost et Jacquet Brocart. Tous deux furent retenus dans le même office au début du principat de Philippe le Bon. Le duc n'était d'ailleurs pas le seul à avoir un garde de la tapisserie et à la fin des années 1390, Guillemot Le Raffle, varlet de chambre et garde de la tapisserie de la duchesse, reçut 30 francs d'or pour les services qu'il avait rendus à Marguerite de $\mathrm{Male}^{78}$.

Certaines responsabilités concernant les chambres, tapisseries et autres produits textiles de luxe associés semblent avoir été de la compétence du

72. - H. VAn DER VELDEN, The Donor's Image: Gerard Loyet and the Votive Portraits of Charles the Bold, Turnhout, 2000, p. 3.

73. - ADCO, B1469, fol. 63.

74. - ADCO, B301, fol. 1.

75. - ADCO, B1860 (portefeuille).

76. - ADCO, B1576, fol. 152.

77. - ADCO, B1514, fol. 65.

78. - ADCO, B1861 (portefeuille). On trouve une situation similaire pour la période suivante où Guillaume de Laire, garde de la tapisserie, travaillait aussi, dans les années 1460, pour Isabelle de Portugal, femme de Philippe le Bon, de même que Garnier Pourcelot, mentionné à la fois comme garde de la tapisserie de la duchesse Isabelle et de son fils Charles le Téméraire dans les années 1470. J. Chipps-Smith, "The Practical Logistics of Art: Thoughts on the Commissioning, Displaying and Storing of Art at the Burgundian Court », dans In detail: new studies of Northern Renaissance art in honor of Walter S. Gibson, L.S. Dixon éd., Turnhout, 1998, p. 27-48. 
garde de la tapisserie. Ce dernier intervenait, en particulier, lorsque les tentures ducales devaient être transportées et installées dans une résidence princière pour une occasion importante ${ }^{79}$. En 1390, Jean Cosset prépara et accompagna le transport de la vaisselle et des tapisseries ducales pour la visite de Charles VI à Dijon ${ }^{80}$. Pierre de Beaumez fut également récompensé « pour ses bons et agréables services » rendus en entreprenant plusieurs voyages pour préparer des tapisseries pour l'hôtel de Conflans en $1394^{81}$. Les réparations faisaient également partie des fonctions qui pouvaient être confiées au garde de la tapisserie, et elles n'étaient pas limitées aux textiles mais pouvaient concerner les autres produits de luxe dont la garde lui incombait. En 1387, Jean Le Cambier, varlet de chambre et garde de la tapisserie, fut payé pour la réparation d'un grand récipient en argent qui fut lavé et poli ${ }^{82}$. Outre l'entretien et la réparation, les voyages destinés à permettre au duc de se fournir en produits textiles faisaient aussi partie des activités du garde de la tapisserie: en 1394, Jacquet Dourdin fut envoyé de Paris à Boulogne par ordre de Philippe le Hardi pour superviser l'achat de deux tappis et les rapporter à Paris ${ }^{83}$.

La nécessité dans laquelle se trouvaient les tapissiers d'entretenir des réseaux en milieu urbain et en milieu aulique, d'exercer plusieurs activités professionnelles et, si possible, de s'assurer d'une position à la cour était en partie due au simple fait qu'être fournisseur de produits textiles de luxe à la cour de Bourgogne impliquait des risques financiers non négligeables. Christopher Dyer a décrit les grands seigneurs comme des clients qui étaient loin d'être fiables, notamment en raison de l'itinérance qui caractérisait leur genre de vie ${ }^{84}$. Même lorsqu'une grande commande ducale était reçue, il n'était nullement garanti qu'une autre allait suivre ni que le fournisseur allait être payé dans des délais raisonnables. Le travail d'Andrée Van Nieuwenhuysen sur les finances de Philippe le Hardi confirme qu'un tel risque était courant pour ceux qui vendaient des produits à la cour ${ }^{85}$. Les paiements pouvaient intervenir trois ou quatre ans après la commande, et même parfois dix ans après ${ }^{86}$. Amelot Dourdin, veuve du tapissier Jacquet Dourdin, fut payée en 1411 pour la livraison de deux tappis armoriés ornés de la devise de Marguerite de Bavière, femme de Jean sans Peur, qui avaient été comman-

\footnotetext{
79. - Ibid., p. 33.

80. - ADCO, B1481, fol. 27 et BNF, Bourgogne 22, fol. 47.

81. - ADCO, B1503, fol. 109.

82. - ADCO, B1466, fol. 11-12.

83. - ADCO, B1503, fol. 127.

84. - C. DYER, «The consumer and the market in the later middle ages », Economic History Review, t. 42,1989 , p. $305-327$.

85. - A. Van Nieuwenhuysen, Les finances du duc de Bourgogne, Philippe le Hardi (1384-1404), Économie et Politique, Bruxelles, 1984, p. 398.

86. - Ibid.
} 
dés en 1402, à l'époque où cette princesse était encore comtesse de Nevers, comme le compte le mentionne ${ }^{87}$.

Une autre grande et coûteuse tenture qui fit courir d'importants risques financiers au tapissier impliqué dans sa vente fut celle de l'Apocalypse ${ }^{88}$. En 1386, Robert Poinçon fut payé 1167 francs pour fournir 100 livres de fil d'or utilisées dans la réalisation de la tapisserie, soit l'équivalent de 2000 journées de travail d'un maître maçon ${ }^{89}$. On trouve trace d'un second paiement lié à cette commande en 1387, Robert Poinçon recevant 1200 francs sur une somme totale de 5000 francs d'or ${ }^{90}$. C'est l'un des plus importants paiements effectués pour un achat de tapisserie durant les principats de Philippe le Hardi et Jean sans Peur, la somme représentant l'équivalent de vingt-deux années de salaire d'un maître maçon. Il s'agissait d'un règlement fait pour la livraison de six grands tappis de hautelice en fil d'Arras, représentant l'istoire de l'Apocalypse ${ }^{91}$. Chaque tapis était mentionné comme mesurant 90 aunes carrées, à l'aune de Paris $\left(1,18 \text { x } 90=106,20 \mathrm{~m}^{2}\right)^{92}$. En 1390, un troisième paiement est mentionné, d'un montant de 1000 francs, cette fois pour l'estoffe et façon de certains tapis de hautelice de l'Apocalypse qu'il fait [pour] monseigneur $^{93}$. Apparaît immédiatement le nécessaire investissement, en matériaux et en main-d'œuvre, qui devait être fait par le fournisseur pour la production d'une pièce d'un tel luxe. Je n'ai pas été en mesure de trouver mention d'autres paiements liés à la vente de ces tentures et il est donc difficile de savoir si Robert Poinçon reçût jamais le reste de ce qui lui était dû. Il est certain, en revanche, que Philippe le Hardi eut bien l'usage de ces tapisseries, car on trouve des articles de comptes concernant leur entretien et leur réparation près de dix ans après leur acquisition. Jacquet Dourdin fut chargé du transport, de Paris à Arras, en 1395, de deux tapis de l'Apocalypse avec des tapis représentant les Douze Pairs de France, les Neuf Preux et trois tapis de chapelle $^{94}$. Peut-être en raison de l'usure due à cette utilisation et à ces transports, on voit Jean de Neufport payé pour avoir fourni quarante aunes de large rubans qui devaient être employées par lui pour border neuf tapisseries en la résidence ducale d'Hesdin ${ }^{95}$. Il apparaît clairement que le poids des responsabilités et des risques retombait sur les hommes qui prenaient en charge les grandes commandes ducales et non sur le prince, leur client.

87. - ADCO, B1570, fol. 221.

88. - ADCO, B301 (liasse).

89. - Ibid. Cavallo note que Poinçon était actif à Paris, Arras et Lille. A.S. CAVallo, Medieval Tapestries (cf. supra n. 1), p. 60.

90. - ADCO, B1466, fol. 26-27.

91. - Ibid.

92. - Ibid.

93. - ADCO, B1495, fol. 72.

94. - ADCO, B1501, fol. 74.

95. - ADCO, B1511, fol. 114. Les rubans étaient des bandes d'étoffe plus ou moins larges qui pouvaient être utilisés pour border ou orner des tapisseries. F. GODEFROY, Dictionnaire de l'ancienne langue française et de tous ses dialectes du IXe au XV siècle, vol. 7, Paris, 1880-1902, p. 258. 
Étant donné les risques financiers que pouvait impliquer une commande passée par la cour ducale, il n'est pas surprenant que les tapissiers de Philippe le Hardi et Jean sans Peur aient été étroitement liés au processus de production nécessité par la réalisation de la commande. Ils étaient, après tout, des professionnels ambitieux, soucieux de survivre sur un marché concurrentiel et de protéger leur réputation. Pierre de Beaumez, l'un des principaux fournisseurs de tapisseries de la cour, paraît avoir été étroitement impliqué dans différentes étapes de l'acquisition et la production de tapisseries. Un document comptable daté de 1398 mentionne un paiement de 20 écus d'or fait aux varlés tapissiers de Pierre de Beaumez, varlet de chambre et tapissier de mondit seigneur pour son vin, en récompense du fait d'avoir œuvré et aidé à l'acquisition du tapis de l'histoire de Fama, « que mondit seigneur a ordonné être faite par ledit Pierre ${ }^{96}$. Cette somme représentait l'équivalent de 90 jours de travail d'un maître maçon brugeois, toutefois, on ne sait pas entre combien de valets la somme fut répartie. Un an plus tard, on trouve une autre référence confirmant le fait que Pierre de Beaumez tenait plusieurs apprentis à son service. À cette occasion, il fut payé pour ses gages et les dépenses d'un valet et deux chevaux, frais nécessaires pour un voyage de Paris à Compiègne effectué sur ordre du duc afin d'y apporter un drap de hautelice représentant l'histoire du Roman de la Rose que le duc de Bourgogne voulait offrir au duc de Berry ${ }^{97}$. Le montant de leurs gages était à peu près équivalent à ce qu'un maître maçon brugeois aurait reçu, soit 10 gros $2 / 3$ par jour. Les varlés de Pierre de Beaumez étaient toujours en service en 1410, date à laquelle un compte mentionne un paiement pour tapis, tapis de chapelle et quarreaux pour Catherine de Bourgogne, fille de Jean sans Peur. Comme à l'accoutumé pour ce type de personnages, ils reçurent une gratification en vin: pour leur vin d'avoir ouvré en ycelle tapisserie ${ }^{98}$.

Pour avoir d'autres informations sur les valets travaillant pour nos fournisseurs, il faut se tourner vers la comptabilité d'autres princes, en l'occurrence vers celle du duc d'Anjou en 1379. Dans un cas semblable à celui de 1398, Nicolas Bataille, «bien qu'appelé Colin dans ce compte » fut chargé, par ordre du duc, de distribuer aux valets de Robert Poisson, « probablement Poinçon », qui avait œuvré aux tapisseries du prince, la somme de 20 francs pour leur vin 99 . Deux ans après la commande des tentures de l'Apocalypse, il est clair que ces personnages étaient étroitement liés à leurs ouvriers et assuraient la productivité de leur force de travail.

\footnotetext{
96. - ADCO, B1517, fol. 196. Pierre de Beaumez fut payé 3000 écus d'or pour trois tapisseries représentant l'histoire de Fama en 1399. Il est possible que la mention citée ici fasse référence à ces tentures. ADCO, B1519, fol. 232-234.

97. - ADCO, B1469, fol. 26.

98. - ADCO, B1560, fol. 170.

99. - W.G. ThOMPSON, A History of Tapestry (cf. supra n. 33), p. 65.
} 
Colart d'Auxy et Jean Armory apparaissent également comme ayant été en mesure d'effectuer des réparations sur les tapisseries ducales avec des varlés à leur disposition. En 1389, Colart d'Auxy, tapissier demourant a Arras, fut payé pour son salaire et les dépenses de son varlet qui, le fait est noté, l'avait assisté dans le travail de réparation de plusieurs grands tapis de sale de chambre en la résidence ducale d'Hesdin ${ }^{100}$. On peut ajouter que Jean Armory, tapissier demeurant à Arras, fut payé, en 1397, pour ses gages et ceux de son varlet ouvrier avec lequel il avait travaillé pour réparer une tapisserie pendant vingt jours. Pour ce travail, ils reçurent 7 s. 6 d. t. par jour, soit quatre gros de moins que les gages moyens d'un maître maçon brugeois ${ }^{101}$. Il fallut une période nettement plus longue à Jean de La Croix, tapissier de Paris, et à son varlet pour effectuer des réparations sur des tapisseries et des chambres de l'hôtel de Conflans et de l'hôtel d'Artois, du 3 décembre 1396 au 2 juin $1397^{102}$. Pour ce travail, Jean de La Croix et son varlet furent payés exactement au même tarif journalier que Jean Armory et son varlet ouvrier. Les sommes versées au titre de leurs gages à ces personnages suggèrent qu'ils étaient des maîtres artisans qui avaient la possibilité de s'adjoindre l'aide de varlés lorsqu'ils étaient chargés de la réparation ou de la finition de tapisseries pour la cour ducale.

Il est essentiel de considérer les tapissiers étudiés dans cet article comme des personnages actifs à la fois dans un cadre urbain et dans le cadre de la cour. S'établir dans les milieux auliques en tant que fournisseurs de produits textiles de luxe était important pour eux dans la mesure où ce fait représentait une étape pour leurs ambitions financières et sociales. Mais fournir la cour n'était pas une fin en soi. Les individus étudiés ici étaient habiles à se constituer des réseaux et des alliances avec d'autres professionnels bien établis et ayant accès à d'autres entreprises commerciales lucratives. Ces réseaux et ces alliances permettaient de limiter les risques financiers liés à une grosse commande ducale, et ces risques financiers expliquent pourquoi, même des fournisseurs réguliers de la cour, contrôlaient de près le suivi des commandes et l'utilisation de la force de travail. De plus, étant donné que les commandes ducales ne suffisaient pas à la majorité des tapissiers mentionnés dans les comptes pour faire vivre leur entreprise, il semble que c'est la réputation professionnelle qui était la valeur la plus importante pour eux. Fournir la cour,

100. - ADCO, B1519, fol. 239. Il reçut 40 fr. 2 s. 6 d. t., l'équivalent de 64 jours de salaire d'un maître maçon; le compte, toutefois ne précise pas le temps passé à Hesdin et ne permet donc pas de connaitre le montant des gages journaliers.

101. - ADCO, B1511, fol. 119.

102. - Ibid., fol. 109. 
même une seule fois, en produits textiles de luxe, contribuait sans nul doute à accroître le prestige de leur production aux yeux de clients potentiels et renforçait leur position sociale dans la ville ${ }^{103}$.

Mots-clés : tapisserie, Arras, cour de Bourgogne, Philippe le Hardi, Jean sans Peur, élites urbaines.

103. - Traduction par Bertrand Schnerb. 
Wilson 6/06/11 16:52 Page 32 